\title{
MODELLING AND ALGORITHMS FOR LOCATION PROBLEMS
}

\section{Christiane Tammer, Martin-Luther-Universität Halle-Wittenberg}

This paper deals with several kinds of location problems, necessary optimality conditions and algorithms based on the optimality conditions.

We will study some generalizations of Fermat-Weber location problem, at first the following convex vector-valued $\mathrm{L}_{\mathrm{p}}$-Approximation problem:

$$
f(u)=\left(\begin{array}{c}
\left\|\sum_{j=1}^{n} u_{j} f_{j}{ }^{1}-f_{o}{ }^{1}\right\| \\
\left\|\sum_{j=1}^{n} u_{j} f_{j}^{m}-f_{o}^{m}\right\|
\end{array}\right] \quad \rightarrow \quad v-m i n
$$

with $\mathrm{f}_{j}^{\mathrm{i}} \in \mathrm{L}_{\mathrm{p}}(\mathrm{S}), \mathrm{f}_{\mathrm{o}}^{\mathrm{i}} \in \mathrm{L}_{\mathrm{p}}(\mathrm{S}), 1 \leq \mathrm{p} \leq \infty,[\mathrm{i}=1, \ldots, \mathrm{m}], S \subset \mathrm{R}^{q}$ compact, $\mathrm{U}=\mathrm{R}^{\mathrm{n}}$.

Since the vector optimization problem (P1) is convex, we can use a scalarization with linear continuous functionals. Applying a variational principle we get necessary conditions for approximately efficient points of (P1) by using the subdifferential calculus. This necessary conditions we will use in order to derive a proximal point algorithm for the vector optimization problem (P1).

Further, we will discuss a population model, which can be described as a nonconvex vector-valued location problem. So we can't apply the results of convex analysis. We will show relations between the efficient solutions of the vector optimization problem and a surrogate scalar optimization problem. By using the directional derivative of the objective functions and a variational principle we derive necessary conditions for approximately efficient solutions. 